\title{
Uneven decline in food system inequality
}

\author{
An assessment of global inequality in agriculture, food and health indicators between 1970-2010 reveals that \\ significant progress has been made in some countries, but more is needed to achieve a truly equitable food system \\ that delivers for the diets, nutrition and health of all people.
}

\section{Shauna M. Downs and Elizabeth L. Fox}

M alnutrition and dietary risk factors are among the leading contributors to the global burden of disease $\mathrm{e}^{1,2}$, with many countries experiencing the co-existence of undernutrition as well as overweight, obesity and diet-related diseases ${ }^{2}$. These burdens of malnutrition disproportionately affect low-income populations around the world, leading to significant nutrition and health disparities ${ }^{3}$.

While there is no shortage of global goals aimed at reducing malnutrition and poor-quality diets, such as the Sustainable Development Goals and World Health Assembly targets, the world is largely off track to meet them ${ }^{4}$. Food systems that fail to provide all people with healthy, safe, affordable and culturally appropriate, sustainable diets are a common denominator of malnutrition globally ${ }^{5}$.

In this issue of Nature Food, Masters and colleagues investigate inequality in key food system input, output and outcome indicators across countries over time ${ }^{6}$. Based on publicly available data sources, they combine Lorenz curves - which enables them to calculate Gini coefficients - and parade plots to measure population-weighted inequality between countries, for food system indicators, using a standardized scale.

The analysis reveals declines in between-country economic inequality between 1970-2010, assessed using gross domestic product. While these analyses demonstrate improvements in economic inequality at the global level, income inequality as well as other forms of inequality remain very high. At the same time, within-country inequality is currently higher than it was 25 years ago 7 and continues to increase in most countries, further exacerbating diet and nutrition disparities and often leading to broader social, economic and political instability ${ }^{8}$. This inequality is intensified by shocks, such as the COVID-19 pandemic and more frequent climate emergencies, that have cascading effects throughout the food system - making it harder for low-income populations to eat well.

The majority of the world's poor live in rural areas, with many engaging in the agricultural sector as a major source of livelihoods ${ }^{9}$. While agricultural growth has the potential to help reduce poverty in rural areas, inequalities in access to resources such as land, inputs and irrigation can thwart progress towards poverty elimination ${ }^{7}$. Masters and colleagues found little, and uneven, progress in terms of reducing global inequality in land area harvested and livestock holdings per capita in rural areas over the 40 years examined. For many poor rural households, livestock can be an important source of nutrition but also a safety net against shocks ${ }^{7}$.

One of the more promising findings from their study points to declining inequality in the availability of key foods and nutrients. While inequality in the availability of animal-source foods (ASFs) has declined substantially, inequality remains high with approximately one-fifth

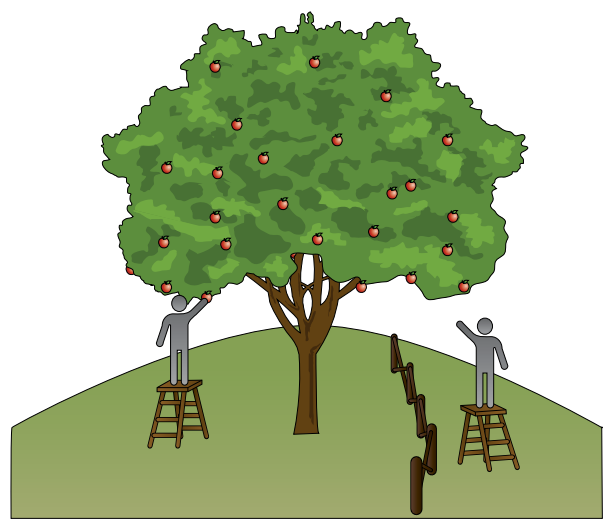

Equality b

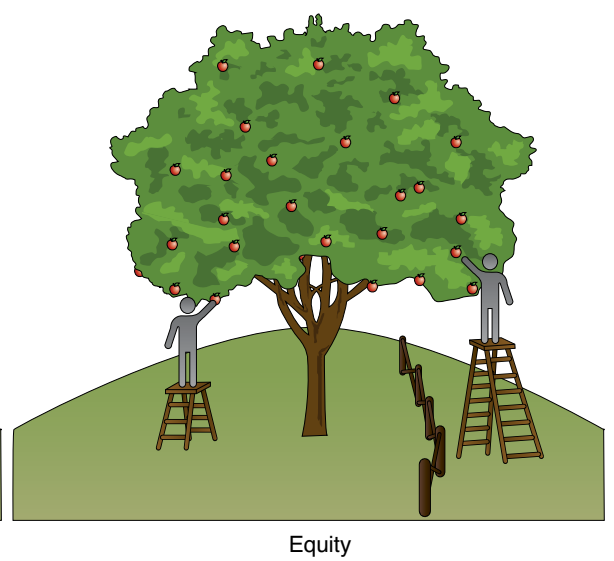

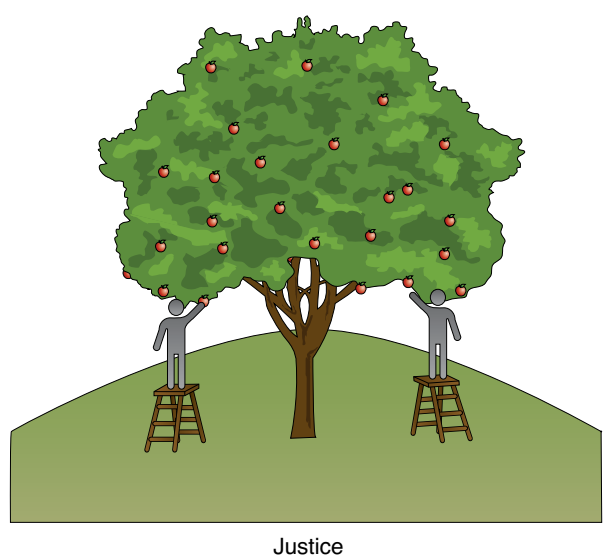

Fig. 1 The difference between equality, equity and justice. a, Equality implies that everyone receives the same resources and support irrespective of their needs and the structural barriers they face. $\mathbf{b}$, Equity implies that individuals receive different resources and support based on their needs, enabling them to have equal access and opportunities. c, Justice implies that structural and systemic barriers are removed allowing individuals to have equal access and opportunities. Figure inspired by ref. ${ }^{15}$. 
of the global population consuming less than $10 \%$ of their energy from ASFs. ASFs can be critical sources of key nutrients, particularly for young children ${ }^{10}$, but high consumption of some foods (for example, red meat) is associated with increased greenhouse gas emissions, freshwater use and loss of biodiversity, as well as diet-related non-communicable diseases (NCDs), particularly if highly processed ${ }^{11}$. Increasing ASF consumption among the lowest-income consumers, and decreasing consumption among the highest, may lead to significant improvements in both health and environmental outcomes ${ }^{11}$.

The authors also observed declines over time in the inequality of fruit and vegetable as well as vitamin $\mathrm{A}$, zinc and iron availability. Moreover, they rightly point out that these results fail to account for differences in iron and zinc bioavailability and absorption, potentially masking inequalities in diet quality. This is a real concern given the much higher levels of inequality noted for iron and zinc from animal sources as compared to plant sources.

However, global analyses such as this one often rely on Food and Agriculture Organization data on the availability of different foods and nutrients as a proxy for dietary intakes, given the dearth of high-quality global dietary data. Additionally, besides being available within the food environments that consumers interact with, nutritious food must also be affordable and acceptable. Thus, by focusing the analyses solely on the availability of these foods and the nutrients within them, inequality might be further underestimated, particularly given the price differential for nutrient-rich foods ${ }^{12}$.
Having access to nutritious foods that are available, affordable and acceptable, while also limiting access to energy-dense foods of low nutritional quality, can help to improve nutrition and health outcomes. Masters and colleagues found marked reductions in the prevalence of undernutrition (that is, stunting and underweight) globally; however, progress towards reducing inequality in nutrition outcomes was uneven. Inequality in the prevalence of stunting and underweight increased globally, while there was convergence and more equality in the prevalence of overweight, obesity and diet-related NCDs (with the exception of diabetes among women). These changing burdens of malnutrition underscore the need to ensure that food environments, as well as the upstream food system drivers and food supply chains that feed into them, promote healthy diets. Double-duty actions that target the underlying drivers of all forms of malnutrition simultaneously are also needed.

The analysis by Masters and colleagues provides insight into convergence in equality of food systems indicators globally, but more granular, disaggregated data is needed to understand the true extent of inequality, as well as inequity, within our food systems. There are numerous structural and systemic barriers across the food system that perpetuate inequality ${ }^{13}$, and which require appropriate tools and changes for the system to become equitable and just (Fig. 1).

Disaggregated, subnational data can help to identify different needs and inequities for different food systems indicators ${ }^{14}$. Such data can also inform policies and interventions to improve diets and reduce the multiple burdens of malnutrition in a way that is tailored to the needs and contexts of a given population, and that eliminates the structural barriers that impede achievement of healthy diets for all.

Shauna M. Downs ${ }^{1 凶}$ and Elizabeth L. Fox (D)2 ${ }^{1}$ Department of Urban-Global Public Health, Rutgers School of Public Health, Newark, NJ, USA. ${ }^{2}$ Master of Public Health Program, College of Veterinary Medicine, Cornell University, Ithaca, NY, USA.

$凶_{e-m a i l: s d 1081 @ s p h . r u t g e r s . e d u}$

Published online: 19 March 2021

https://doi.org/10.1038/s43016-021-00247-3

References

1. Murray, C. J. et al. Lancet 396, 1223-1249 (2020).

. Swinburn, B. A et al Lancet 393, 791-846 (2019).

3. Perez-Escamilla, R. et al. BMJ 361, k2252 (2018).

4. Tracking Progress on Food and Agriculture-related SDG Indicators 2020: A Report on the Indicators Under FAO Custodianship (FAO, 2019).

5. Branca, F. et al. Lancet 395, 8-10 (2020).

6. Bell, W., Lividini, K. \& Masters, W. A. Nat. Food https://doi. org/10.1038/s43016-021-00241-9 (2021).

7. De La O Campos, A. P., Villani, C., Davis, B. \& Takagi, M. Ending Extreme Poverty in Rural Areas: Sustaining Livelihoods to Leave No One Behind (FAO, 2018).

8. World Social Report 2020: Inequality in a Rapidly Changing World (United Nations, 2020).

9. Townsend, R. F., Ceccacci, I., Cooke, S., Constantine, M. \& Moses, G. Implementing Agriculture for Development: World Bank Group Agriculture Action Plan (2013-2015) (World Bank Group, 2013).

10. Raiten, D. J. et al. Curr. Dev. Nutr. 4, nzaa087 (2020).

11. Willett, W. et al. Lancet 393, 447-492 (2019).

12. Headey, D. D. \& Alderman, H. H. J. Nutr. 149, 2020-2033 (2019).

13. Botreau, H. \& Cohen, M. J. Adv. Food Secur. Sustain 5 53-117 (2020)

14. Hawkes, C., Fox, E., Downs, S. M., Fanzo, J. \& Neve, K. Glob. Food Sec 27, 100414 (2020).

15. Ruth, T. A visual summary. In Design in Tech Report 2019: Section 6: Addressing Imbalance (11 March 2019); http://go.nature. com/3b56Pfx

Competing interests

The authors declare no competing interests. 